\title{
4 \\ Onsager-molecule approach to screening potentials in strongly coupled plasmas
}

\author{
YAAKOV ROSENFELD \\ Nuclear Research Center - Negev, P.O.Box 9001, Beer-Sheva, Israel
}

\begin{abstract}
The solution of the exact integral equation for the liquid pair-structure in the asymptotic strong coupling limit for the plasma, as mapped on the Onsager charge-smearing optimization for the energy lower bound, features "Onsager atoms" and "Onsager molecules". The universal properties of this asymptotic limit make it a natural reference starting point for an asymptotic strong coupling expansion for the fluid structure and thermodynamics, playing the role of an "ideal liquid" state. In particular, the leading strong coupling terms for the potential energy, direct correlation functions, and screening potentials for the Coulomb and Yukawa mixtures (corresponding to classical plasmas and electron screened classical plasmas), with full thermodynamic consistency, are presented. These are in complete agreement with the Alastuey-Jancovici analysis of early simulations data by Hansen in strong coupling, and with recent highly accurate simulations data of Ogata, Iyetomi, and Ichimaru. Data analysis errors lead Ogata, Iyetomi, and Ichimaru to incorrect results for the short range screening potentials in strong coupling. Their calculations for the short range screening potentials, bridge functions, and enhancement factors for nuclear reaction rates in strongly coupled plasmas, should be revised.
\end{abstract}

La solution de l'équation intégrale exacte pour la structure du liquide dans la limite asymptotique de couplage fort pour le plasma, calquée sur l'optimisation de charge d'Onsager pour la limite inférieure de l'énergie, met en évidence des "atomes d'Onsager" et des "molécules d'Onsager". Les propriétés universelles de cette limite asymptotique en font un point de réf'erence naturel pour un développement asymptotique en couplage fort pour la structure et la thermodynamique du fluide. En particulier, les ter- 
mes dominants en couplage fort pour l'énergie potentielle, les fonctions de corrélation directes, et les potentiels d'écran des mélanges de Coulomb et de Yukawa (correspondant aux plasmas classiques et aux plasmas classiques écrantés), sont pr'esentés dans cette revue. Ces résultats sont en parfait accord avec l'analyse de Alastuey et Jancovici des premières simulations de Hansen en couplage fort, et avec les récentes simulations splus précises de Ogata, Iyetomi et Ichimaru. Des erreurs d'analyse de données ont conduit Ogata, Iyetomi et Ichimaru à publier des résultats incorrects pour la partie à courte portée des potentials d'écran en couplage fort. Leurs calculs pour les potentiels d'écran, les fonctions bridge, et les facteurs d'accroissement des taux de réactions nucléaires dans les plasmas fortement couplés doivent être revus.

\subsection{Introduction : screening potentials as related to the inverse scattering problem for the fluid pair structure}

Classical plasmas composed of classical positive ions in a uniform neutralizing background charge density of degenerate electrons, are basic models for dense stellar materials and provide important reference systems in condensed matter physics (Rogers \& DeWitt (eds.), 1987; Ichimaru (ed.), 1990). These systems are referred to as "one component plasma" (OCP), "binary ionic mixture" (BIM), or "multi ionic mixture", depending on the number of different ionic species. Considerable effort has been invested over the years (Hansen \& Baus, 1980; Ichimaru 1982; Ichimaru et al., 1987; Isern, these proceedings) in increasing the accuracy of the calculations of the short range screening potentials in strongly coupled plasmas because of the following two main reasons: (1) The enhancement factors for the thermonuclear reaction rates which are important for stellar evolution, particularly for Carbon ignition in degenerate cores, are essentially controlled by the short range part of the screening potential, (the zero-separation value, $H(0)$, in particular). The ions inside dense stars (such as white dwarfs and neutron stars) are strongly coupled,$\Gamma \sim($ potential energy)/(kinetic energy) $\sim 100$, the enhancement factor is roughly proportional to $\exp [H(0)]$, and $H(0) \sim \Gamma$, so that an error of $2 \%$ in the value of $H(0)$ may yield a reaction rate which is off by an order of magnitude. (2) The screening potentials play a key role in the study of the short range behavior of the bridge functions, notably their universal properties, which proved seminal for developing an accurate theory of liquid structure (Rosenfeld \& Ashcroft, 1979; Rosenfeld 1980a,b). The zero separation theorem (Hoover \& Poirer, 1962; Widom, 1963; Rowlinson \& Widom, 1982) provides an important test for the accuracy of the 
equation of state of mixtures, and offers a consistency check (Rosenfeld, 1990a) for predictions based on closure approximations in integral equation theories for the fluid pair structure.

The screening potentials $H_{i j}(r)$ of the classical plasma are defined in terms of the bare Coulomb interaction between the two ions of charges $Z_{i}$ and $Z_{j}$, and the pair correlation function $g_{i j}(r)$ :

$$
H_{i j}(r)=\frac{Z_{i} Z_{j} \Gamma}{r}+\ln \left[g_{i j}(r)\right]
$$

It is convenient to measure all distances in units of the Wigner-Seitz radius, $a=\left(\frac{3}{4 \pi n}\right)^{1 / 3}$, where $n$ is the total number density of the ions, and to define the Coulomb coupling parameter $\Gamma=\frac{e^{2}}{a k_{B} T}$. The pair correlation function can be expressed through the free energy change upon fixing the positions of the pair of fluid particles in the appropriate configuration to form an interaction-site molecule (Hoover \& Poirer, 1962; Widom, 1963; Jancovici, 1977; Rosenfeld, 1987a,b)

$$
H_{i j}(r)=-\frac{\left\{F_{1}^{e x}(r)-F_{0}^{e x}\right\}}{k_{B} T}+\frac{Z_{i} Z_{j} \Gamma}{r}
$$

Here $F_{0}^{e x}$ is the configurational (excess over ideal gas) free energy of the Nparticle system (in a uniform neutralizing background) and $F_{1}^{e x}(r)$ is that of the same system but with the pair of particles kept at fixed separation, $r$ , forming a two-site charge cluster. $F_{1}^{e x}(r)$ does contain the intramolecular interaction $\frac{Z_{i} Z_{j} \Gamma}{r}$, so that $H_{i j}(r)$ is finite as $r \rightarrow 0$.

The simulation data is limited to $r<R_{\min }$ where $R_{\min } \sim 1$ for $\Gamma \sim 160$ and $R_{\min } \sim 0.5$ for $\Gamma \sim 10$. It is impossible to get $H(r \sim 0)$ by simulations directly from eq.(1) because of the essentially zero probability for very close encounters. It is possible to try and extrapolate these results down to $r=0$, but early attempts were not very successful (DeWitt, Graboske, and Cooper, 1973; Itoh, Totsiji, Ichimaru, and DeWitt,1978,1979) as pointed out by Rosenfeld (1980a, 1987a,b). On the basis of eq.(2), however, the calculation of $H(0)$ can be carried out via the equation of state of the plasma mixture, if it is available. Salpeter (1954) and Salpeter \& Van Horn (1969) pioneered the calculation of eq.(2) near $r=0$ by means of the ion-sphere model. Using eq.(2) and assuming the validity of the linear mixing rule (Hansen et al., 1977; Brami et al., 1979) for the free energy of the BIM and by employing an accurate OCP equation of state (DeWitt, 1976), Jancovici (1977) obtained a good estimate of $H(0)$ for the OCP. He also derived the exact leading $r^{2}$ term in the expansion around $r=0$, which was used by 
Alastuey and Jancovici (1978) to extrapolate the simulation data for $H(r)$ towards the origin $r=0$.

The first direct (structural, via eq.1) theoretical calculations of $H_{i j}(r)$ for the OCP and the BIM were performed by the assumption of universality of the bridge functions in the modified-hypernetted-chain (MHNC) theory (Rosenfeld, 1980a,b). These results are in agreement with Jancovici's calculations as well as with the ion-sphere scaling for mixtures. The classical direct $(\phi(r) \rightarrow S(k))$ and inverse $(S(k) \rightarrow \phi(r))$ problems for liquid pair structure, relating the structure factor, $S(k)$, to the pair potential, $\phi(r)$, can be reduced by exact diagrammatic analysis to the solution of the hypernetted-chain (HNC) integral equation for an effective potential (denote: $\beta=\frac{1}{k_{B} T}$ )

$$
\Phi(r)=\phi(r)+\frac{B(r)}{\beta}
$$

The "exact" HNC equation is composed of the Ornstein-Zernike relation between the direct correlation function, $c(r)$, and the radial distribution function $g(r) \equiv h(r)-1$, with the $k$-space form (also defining the structure factor $S(k)$ )

$$
h(k)=c(k)+n h(k) c(k) \equiv S(k) c(k)
$$

and the HNC-closure for $\Phi(r)$ :

$$
H(r)-\beta \phi(r) \equiv \ln [g(r)]=-\beta \Phi(r)+h(r)-c(r)
$$

$n=N / V$ is the number density. The heart of the problem is $B(r)$ - the bridge function - which may be expanded in diagrams with the "dressed" $h(r)$-bond. $B(r)=0$, i.e. $\Phi(r)=\phi(r)$, defines the HNC-approximation. The diagrammatic low density expansion represents a very slowly convergent route for obtaining meaningful results for a highly correlated system like a dense fluid, while, on the other hand, even the HNC-approximation provides an excellent point of departure for describing liquid pair structure. Along this alternative route, a first order improvement on the HNCapproximation, the ansatz of the universality (Rosenfeld \& Ashcroft, 1979) of the repulsive short range structure of $B(r)$ was found empirically to be very accurate. It provides the key (Rosenfeld, 1986a; Aers \& Dharmawardana, 1984) to consistent solutions of the direct and inverse "scattering problems". Heuristic arguments were given in favor of "universality", but 
the computational intractability of the bridge diagrams prohibited its direct assessment. More recently, the "Onsager molecule" method (Rosenfeld, $1987 \mathrm{a}, \mathrm{b}$ ) was advanced for the evaluation of eq.(2). It is based on a general result in liquid state theory (Rosenfeld 1985,1986b), which holds under broad assumptions, that the leading term in the asymptotic strong coupling expansion of the configurational free energy is also its Onsager-type exact lower bound, e.g. the ion-sphere result for the classical plasmas. Using the Onsager-Molecule approach we can calculate the leading strong coupling expansion term of $H(r)$ (Rosenfeld, 1988, 1991a; Rosenfeld, Levesque, and Weis, 1989) and from it derive the asymptotic $B(r)$, closing a fully self consistent cycle. The results using this method (Rosenfeld, 1992a) are in agreement with Jancovici's expression, with the MHNC calculations, and with the simulation results, as will be described below.

Very recently, using an extrapolation of very accurate "extra long" computer simulations for strongly coupled classical plasmas, new results for the short range screening potential $H(r)$ were obtained by Ogata, Iyetomi and Ichimaru (1991). These results are reported to be of $0.1 \%$ accuracy and to differ significantly from previous calculations (e.g. Jancovici's expression (1977) for $H(0)$ ). Careful analysis of the same data finds (Rosenfeld, 1992a), however, that these new simulations data are in excellent agreement with the previous calculations. Moreover, these high accuracy simulations provide an additional strong support to the asymptotic expansion ("Onsager molecule") analysis (Rosenfeld, 1987a,b) of short range screening potentials in multi-ionic plasmas. It is mainly their incorrect data analysis, which led Ogata, Iyetomi and Ichimaru (1991) to wrong conclusions. The analysis by Ogata, Iyetomi and Ichimaru (1991) is also used as the lounching platform for similar calculations of electronic-response effects by Ichimaru and Ogata (1991) and for general multi ionic mixtures by Ogata, Ichimaru and Van Horn (1991). The errors in these later calculations were also pointed out (Rosenfeld, 1992a). A very recent review by Ichimaru (1993), summarizes the many papers on screening potentials and enhancement factors for nuclear reaction rates by Ichimaru and coworkers, which perpetuate these errors. Although mentioning this criticism, the review by Ichimaru does not present any serious attempt to address it. In turn, the difference of results from the Ichimaru group $v s$ the older Jancovici-Alastuey work could make a difference of a massive accreting white dwarf exploding into a Type I supernova or collapsing into a neutron star (Isern, these proceedings). The present review was invited in order to clarify the situation.

This review describes the properties of strongly coupled plasmas as obtained analytically from the Onsager-molecule theory in complete agreement 
with the numerical simulations data. The Onsager molecule theory provides the asymptotic strong coupling properties of classical plasmas in a simple physically intuitive way, and is an essential tool for analysing numerical simulation results for strongly coupled plasmas. Ogata, Iyetomi and Ichimaru completely ignore the asymptotic analysis, which could have provided them an early worning about their wrong assumption. Along with revealing the errors in the analysis by Ogata, Iyetomi and Ichimaru, this review proposes a revised calculation of the short range screening potentials from their accurate simulations data.

\subsection{Asymptotic strong coupling limit for plasmas (Onsager atoms and molecules) as a paradigm for the "ideal liquid" state}

Unlike the solid, on one hand, and the ideal gas, on the other hand, the dense fluid near freezing does not contain a "natural" small parameter. The solid features the crystal structure as the "ideal" reference state; the Lindemann melting rule identifies the ratio of particle's vibration to the nearest neighbor distance as a natural small parameter; the "elementary excitations" are phonons, providing the basis for the harmonic expansion. The low density fluid features the uniform distribution "ideal gas" as the reference state, with a natural small parameter given by the particle's size to the WignerSeitz radius. The "elementary excitations" are pair-collisions, and the virial power series in density is the natural expansion (for plasmas consider the Debye theory and the Abe-Meeron cluster expansion). There is, however, no natural "ideal liquid" available, but it can be theoretically constructed by using the asymptotic high density limit (bypassing the solid) of the exact diagramatic theory of liquids, to have all the needed properties in order to serve its purpose (Rosenfeld, 1985,1986b,1991a). This ideal liquid features universal structure-factor peaks, e.g. at $\operatorname{tg}\left(k_{i} a\right)=k_{i} a$ for $D=3$. In parallel it features "Onsager atoms" with self energy that provides an exact energy lower bound. The small parameter is a generalized free-volume, the "elementary excitations" are "Onsager molecules", and the "basis functions" are the fundamental geometric measures of the sphere. The "ideal liquid" is defined through mapping of the asymptotic high density or strong coupling limit of the exact liquid state theory for pair-correlations onto the Onsager exact lower bound for the potential energy (the corresponding variational problems coincide). It is extended by recursive definitions for higher order correlations and provides basis functions for fluid structure and free energy functional (Rosenfeld, 1993a). 
Exact liquid state theory for pair correlations can be reduced to an HNC equation for some potential and thus general properties of the solution of this equation for different potentials are of central importance. For any non-singular potential $\Phi(r)$ with strong repulsion at short distances, the asymptotic $\Gamma \rightarrow \infty$ solution of the HNC equation has the following universal features (Rosenfeld, 1988) ( use $a\left(=\left(\frac{3}{4 \pi n}\right)^{1 / 3}\right.$ in $3 D$ ) as the unit of length, and $\beta \Phi(r)=\frac{\Gamma}{r}$ for the OCP in $3 D$ as the specific example) : (1) Madelung behavior of the potential energy featuring: the Onsager exact lower bound for the potential energy = sum of self energy of individual dressed particles (Onsager atoms and molecules), e.g. for the OCP

$$
\frac{\beta U}{N}=\beta u_{O A}=-0.9 \Gamma,
$$

$u_{O A}$ is the self-energy of an "Onsager atom" consisting of a point charge at the center of a neutralizing unit sphere having the background charge density. It is equal to the energy integral with a universal pair correlation function

$$
\begin{aligned}
\frac{\beta U}{N} & =\frac{1}{2} n \int[g(r)-1] \beta \phi(r) d^{D} r \\
& =\frac{1}{2} \Omega_{D}^{-1} \int\left[g_{D}(r)-1\right] \beta \phi(r) d^{D_{r}}
\end{aligned}
$$

(in our units $\mathrm{n}=\Omega_{D}^{-1}$, where $\Omega_{D}$ is the volume of a unit D-dimensional sphere). The universal functions $g_{D}(r)$ denote the limit $\eta \rightarrow 1$ of the solution of the PY equation for D-dimensional hard spheres of packing fraction $\eta$. (2) Saturation of the direct correlation functions: $-\frac{c(r)}{\Gamma}=\Psi(r)=$ electrostatic interaction between the uniformly smeared charges (spheres with the ionsphere radius), for the $\mathrm{OCP}$, given in $3 D$ by

$$
\begin{array}{rlrl}
\Psi(r) & =\frac{6}{5}-\frac{1}{2} r^{2}+\frac{3}{16} r^{3}-\frac{1}{160} r^{5} & r \leq 2 \\
\Psi(r) & =\frac{1}{r} & r & \geq 2
\end{array}
$$

(3) Ewald identity and Mean Spherical Approximation boundary conditions:

$$
\begin{aligned}
\frac{\beta U}{N} & =\frac{1}{2} n \int[g(r)-1] \beta \phi(r) d^{D_{r}} \\
& =\frac{1}{2}\left\{-n \int[c(r)+\beta \phi(r)] d^{D} r+c(r=0)\right\}
\end{aligned}
$$


(a) The direct correlation function is a short range Ewald function: $c(r \geq$ $2)=-\beta \Phi(r \geq 2)$. (b) There exists a positive definite structure factor, $S(k) \geq 0$ : thus also $c(k) \leq 0$. (4) Pair exclusion: $g(r \leq 2)=0$, i.e. $c(r \leq 2)+\beta \Phi(r \leq 2) \geq 0$. The ideal liquid limit is associated with an effective packing fraction $\eta=1$. $\epsilon=1-\eta$ serves as the small parameter. (5) Universal structure factor peaks: Let $\Omega(r)$ denote the overlap volume of two D-dimensional unit spferes at separation $r\left(\Omega(0)=\Omega_{D}\right)$, and define $\omega(r)=\frac{\Omega(r)}{\Omega(0)}$. For $D=3$ obtain

$$
\begin{array}{llrl}
\omega(r) & =1-\frac{3}{4} r+\frac{1}{16} r^{3} & & r \leq 2 \\
\omega(r) & =0 & r \geq 2
\end{array}
$$

The zeroes $c\left(k_{i}\right)=0$ are identical to the zeroes $\omega\left(k_{i}\right)=0$, e.g. $k_{i}=\tan \left(k_{i}\right)$ in $3 D$. For the Coulomb potential $c(k) \propto-\frac{\Gamma \tilde{\omega}(k)}{k^{2}}$, for the Yukawa potential $c(k) \propto-\frac{\Gamma \tilde{\omega}(k)}{k^{2}+\alpha^{2}}$. (6) Correlation functions and screening potentials feature the Onsager molecules naturally and by recursive definition: In the limit $\Gamma \rightarrow \infty$

$$
\begin{aligned}
& F_{0}^{e x}=N u_{O A} \\
& F_{1}^{e x}(r)=(N-2) u_{O A}+u_{O M}(r)
\end{aligned}
$$

and thus from eq.(2)

$$
H_{O M}(r)=\frac{\Gamma}{r}-\beta u_{O M}(r)+2 \beta u_{O A}
$$

Similarly to the Onsager atom, $u_{O M}(r)$ is the self-energy of an "Onsager molecule" consisting of a pair of ions separated by a distance $r$ in a uniform neutralizing charge cloud of background charge density. The shape of this molecule is determined by the surface on which the electrostatic field vanishes. $H_{O M}(r) \leq \frac{\Gamma}{r}$ by virtue of the antibonding of the Thomas-Fermi molecules. Onsager molecules have the property to "dissociate" whenever the distance between the two point charges is larger than $2 a$, i.e.

$$
\begin{aligned}
u_{O M}(r) & =2 u_{O A} \\
\text { i.e. } \quad H_{O M}(r) & =\frac{\Gamma}{r} \quad \text { for } \quad r \geq 2 a
\end{aligned}
$$


Since the Onsager molecule is achieved by optimization,

$$
H_{O M}(r) \leq \Psi(r)
$$

Since

$$
\lim _{\Gamma \rightarrow \infty} \frac{g(r)}{\Gamma}=0
$$

then the asymptotic functions obey

$$
H(r)=-c(r)-B(r)
$$

(7) The asymptotic "energetics" is exact: recall that the energy integral is equal to that with a universal pair correlation function, and change in $\mathrm{B}(\mathrm{r})$ does not affect the asymptotic (Madelung) energy $; H_{O M}(r)$ is the exact asymptotic limit for the screening potential!

\subsection{Exact solution of the classical inverse scattering problem in the strong coupling limit, and asymptotic expansion for the correlations}

We now obtain (Rosenfeld, 1988) the solution of the inverse scattering problem in the ideal liquid limit in $3 D$. Consider the asymptotic HNC limit for $\Phi(r)=\phi(r)+k_{B} T B(r)$, given the Onsager-molecule screening potential $H_{O M}(r)$. Our purpose is to find $\Delta c(r)=c(r)-c_{0}(r)$ and $B(r)$, where $c_{0}(r)$ is the asymptotic HNC limit for $\phi(r)$.

(1) Note that as part of the general "ideal liquid" properties:

$$
\Delta c(r \geq 2)=B(r \geq 2)=0
$$

(2) Because $c(r)$ is an Ewald function corresponding to the Onsager atom we obtain (recall eq.9)

$$
\begin{aligned}
\widetilde{\Delta c}(k) & \leq 0 \\
\Delta c(r=0) & =\frac{3}{4 \pi} \int \Delta c(r) d^{3} r
\end{aligned}
$$

(3) There is only one solution: $\Delta c(r)$ is proportional to the overlap volume function 


$$
\Delta c(r)=-A \omega(r)<0
$$

(4) The coefficient $A>0$ is obtained by requiring thermodynamic consistency between the compressibility and energy equations of state, knowing that the latter is the exact asymptotic limit. For the OCP , $A=0.2 \Gamma$.

(5) Finally, the bridge function is obtained from eq.(15):

$$
B(r)=-\Delta c(r)-c_{0}(r)-H_{O M}(r)
$$

(6) Because of (14a) the asymptotic bridge functions satisfy

$$
B_{O M}(r) \geq-\Delta c(r)
$$

In the range relevant to the scattering problem of strongly coupled plasmas, $r>\sim 1$ ( as $g(r<\sim 1)=0$, in strong coupling), the deviations between $H_{O M}(r)$ and $\Psi(r)$ is less than $1 \%$. However, the corresponding deviations between $B_{O M}(r)$ and $-\Delta c(r)$ are much larger and may exceed $50 \%$ ! The bridge function as obtained from the right hand side of (18a) is universal , i.e. it is of the same shape for all potentials, and equals

$$
B_{\text {universal }}(r)=-\Delta c(r)=A \Gamma \omega(r)
$$

It thus represents the universal component of the strong coupling $B(r)$. $B_{\text {universal }}(r)$ always provides an exact lower bound to the asymptotic bridge function.

Here is a summary of the asymptotic strong coupling limit results for the OCP (Rosenfeld 1988, 1991a): (a) Energy:

$$
\frac{\beta U}{N}=-0.9 \Gamma
$$

(b) Structure: For the short range part (before "dissociation"), $r \leq 2$, we have

$$
\begin{aligned}
-\frac{c(r, \Gamma)}{\Gamma} & =\Psi(r)+\frac{1}{5} \omega(r) \\
\frac{c(0, \Gamma)}{\Gamma} & =-1.4
\end{aligned}
$$




$$
\begin{aligned}
& \frac{H(r, \Gamma)}{\Gamma}=\frac{H_{O M}(r, \Gamma)}{\Gamma} \\
& \equiv \mathcal{H}(r)=\frac{9}{10}\left(2^{5 / 3}-2\right)-\frac{1}{4} r^{2}+h_{2}(r) r^{4} \\
& h_{2}(r) \cong 0.038-0.0026 r^{2} \Rightarrow h_{2}(r=2) \cong 0.0277 \\
& \frac{B(r, \Gamma)}{\Gamma}=\frac{B_{O M}(r, \Gamma)}{\Gamma} \\
& \equiv b(r)=\Psi(r)+\frac{1}{5} \omega(r)-\mathcal{H}(r)
\end{aligned}
$$

$h_{2}(r)$ was obtained by fitting the Onsager molecule numerical data (Stein, Shalitin and Rosenfeld, 1988), while its value at $r=2$ is determined by $\mathcal{H}(r=2)=0.5$. In the longer range part (after "dissociation"), $r \geq 2$, we have

$$
\frac{H(r, \Gamma)}{\Gamma}=\frac{1}{r}=-\frac{c(r, \Gamma)}{\Gamma} ; \frac{B(r, \Gamma)}{\Gamma}=0
$$

The Onsager molecule functions for mixtures, namely $\Psi_{i j}(r)$ and $\omega_{i j}(r)$ are easily obtained analytically as generalizations of the OCP. $H_{O M, i j}(r<$ 2 ) is still needed to be calculated numerically (see, however, the next section). Finally, the ideal liquid Onsager limit and the liquid pair structure belong to the same basin of attraction with respect to the diagramatic iterative map as implied by eqs.3-5. This iterative map exhibits an instability of the pair function which correlates well with the simulation data for the freezing of simple fluids and plasmas. The availability of the Onsager limit result is seminal for these instability calculations (Rosenfeld, 1991b, 1992b).

\subsection{Ion-sphere and Onsager molecule scaling properties for multi-ionic mixtures}

The leading term in the asymptotic strong coupling expansion of the configurational free energy of the fluid is an exact lower bound (Rosenfeld, 1985, 1991a), e.g. the ion-sphere result for the classical plasmas (Lieb and Narnhofer, 1975). The two free energies in eq. 2 are bounded from below by these leading terms. In particular, the excess free energy of the given mixture is bounded by the sum of Onsager-atom self energies: 


$$
\frac{F_{0}^{e x}}{N k_{B} T} \geq \sum_{i} x_{i} u_{O A, i}=-0.9 \sum_{i} x_{i} \Gamma_{i}
$$

$u_{O A, i}=-0.9 \frac{\Gamma Z_{i}^{2}}{R_{i}}=-0.9 \Gamma_{i}$ is the self-energy of an "Onsager atom" consisting of a point charge $Z_{i}$ at the center of a neutralizing sphere of radius $R_{i}$ having the background charge density, $R_{i}=\left(\frac{Z_{j}}{\langle Z\rangle^{1 / 3}}\right.$. Recall that all distances are measured in units of the Wigner-Seits radius. Eq.25 provides the motivation for the linear law. For the mixture composed of charges $Z_{i}$ of relative concentrations $x_{i}$, define $\Gamma_{i}=Z_{i}{ }^{5 / 3}<Z>^{1 / 3} \Gamma$ where $\langle Z\rangle$ is the average charge per ion, then the linear-law reads (Hansen, Torrie, and Vieillefosse, 1979; Brami, Hansen, and Joly, 1979):

$$
\frac{F_{m i x}^{e x}}{N k_{B} T}=\sum_{i} x_{i} f_{0}\left(\Gamma_{i}\right)
$$

where $f_{0}(\Gamma)$ is the OCP excess free energy per particle in temperature units. The screening potential is obtained as a difference between two exact lower bounds, as (Rosenfeld, 1987a,b)

$$
H_{O M, i j}(r)=\frac{Z_{i} Z_{j} \Gamma}{r}-u_{O M, i j}(r)+u_{O A, i}+u_{O A, j}
$$

where $\mathrm{u}_{O M, i j}$ is the self-energy of an "Onsager molecule" consisting of a pair of ions $Z_{i}, Z_{j}$ separated by a distance $r$ in a uniform neutralizing charge cloud of the background charge density. The shape of this molecule is uniquely determined by the surface on which the electrostatic field vanishes.

Onsager molecules as defined have the property to "dissociate" when the distance between the two point charges is larger than $R_{i}+R_{j}$, i.e.

$$
\begin{aligned}
& u_{O M, i j}(r)=u_{O A, i}+u_{O A, j} \quad \text { for } \quad r \leq R_{i}+R_{j} \\
& \frac{H_{O M, i j}(r)}{\Gamma}=\frac{Z_{i} Z_{j}}{r} \quad \text { for } \quad r \geq R_{i}+R_{j}
\end{aligned}
$$

The continuity of the function $H_{O M, i j}(r)$ and its first derivatives implies that near "dissociation" a few-term Taylor series expansion of (28b) around $r=R_{i}+R_{j}$ will provide a good estimate of the function. This Taylor expansion has the following scaling form $\left(d_{i j}=\frac{R_{i}+R_{j}}{2}\right)$ : 


$$
H_{O M, i j}\left(r \leq R_{i}+R_{j}\right)=\frac{Z_{i} Z_{j}}{d_{i j}} H_{O M}\left(\frac{r}{d_{i j}} \leq 2\right)
$$

$H_{O M}(r \leq 2)$ is the corrresponding function for the OCP with $Z_{i}=1$, which is reasonably well represented in the region $1.3 \leq r \leq 2$ by the electrostatic interaction of two uniformly charged spheres of unit radius and unit charge. The region of validity of this scaling approximation, which relates the screening potentials of the mixture to the OCP result, thus covers the region of availability of simulation data $R_{\min } \leq r \leq 2$ in strong coupling (Rosenfeld, 1987a,b). From elementary electrostatics we find that the small- $r$ expansion takes the form

$$
\frac{H_{O M, i j}(r)}{\Gamma}=h_{i j, 0}-h_{i j, 1} r^{2}+\ldots
$$

where

$$
h_{i j, 0}=0.9\left(\frac{\left(Z_{i}+Z_{j}\right)^{2}}{\left(R_{i}^{3}+R_{j}^{3}\right)^{1 / 3}}-\frac{Z_{i}^{2}}{R_{i}}-\frac{Z_{j}^{2}}{R_{j}}\right)
$$

and

$$
h_{i j, 1}=0.5 \frac{Z_{i} Z_{j}}{R_{i}^{3}+R_{j}^{3}}
$$

This coefficient of the $r^{2}$ term as obtained from the Onsager molecule is also the exact term for the plasma mixture, as obtained by Ogata, Iyetomi, and Ichimaru (1991). Comparison of (28) and (29) shows the relevance of two different lengths : $d_{i j}=\frac{R_{i}+R_{j}}{2}$ which is the "dissociation" radius, and $R_{i+j}=\left(R_{i}^{3}+R_{j}^{3}\right)^{1 / 3}$ which is the ion-sphere radius of the molecule at small values of $r$. We need to consider separately the cases of "like" $(i=j)$ and "unlike' $(i \neq j)$ ions.

For "like" $(i=j)$ ions, $d_{i i}=R_{i}$ by definition, and the Onsager-molecule screening potentials obey

$$
H_{O M, i i}(r)=\frac{Z_{i}^{2}}{R_{i}} H_{O M}\left(\frac{r}{R_{i}}\right)
$$

where $H_{O M}(r)$ is the OCP result for $Z_{i}=1$.

For "unlike' $(i \neq j)$ ions there is a cross-over of the relevant scaling length ,as $r$ changes from "dissociation" to "zero separation", between $d_{i j}$ 
and $R_{i+j}$. In view of (28) and (29) then the following scaling approximation can be obtained for the "Onsager molecule" screening potentials of unlike ions:

$$
H_{O M, i j}(r)=G(r) \frac{Z_{i} Z_{j}}{d_{i j}} H_{O M}\left(\frac{r}{d_{i j}}\right)
$$

where the prefactor $\mathrm{G}(\mathrm{r})$ varies slowly between $G\left(r=d_{i j}\right)=1$ and

$$
G(r=0)=G_{0}=\frac{\left(s^{1 / 3}+1\right)\left[(s+1)^{5 / 3}-s^{5 / 3}-1\right]}{4\left(2^{2 / 3}-1\right) s}
$$

where $s=\frac{Z_{i}}{Z_{j}} \geq 1$.

The Onsager molecule results suggest the following general scaling relation for plasma mixtures:

$$
H_{i j}(r)=G(r) H\left(\Gamma_{i j}, \frac{r}{d_{i j}}\right)
$$

where $H(\Gamma, r)$ is the OCP result for $Z_{i}=1, \Gamma_{i j}=\frac{Z_{i} Z_{j} \Gamma}{d_{i j}}$, and where it is expected that $G=1$ for like ions $(i=j)$, and $G=1$ for unlike ions in the region close to $r=d_{i j}$.

\subsection{Electron response corrections to the interionic screening potentials}

The starting point for analysing the electron response corrections to the interionic screening potentials is to consider classical mixtures of charged particles interacting through the repulsive Yukawa (screened Coulomb) potentials (Rowlinson, 1989). The Yukawa intermolecular potential, $\phi(r)=\frac{e^{-a r}}{r}$, has the special property (Rosenfeld, 1993a,b) that it gives rise to the same functional form for the potential outside a spherically symmetric distribution of matter : Thus, if a point Yukawa charge $Z$ is smeared out radially with distribution $\rho(r)$ upto a finite radius $R$, and if at the same time the charge is appropriately renormalized, $Z_{\text {renorm }}=q(\alpha, R) Z$,

$$
\frac{1}{q(\alpha, R)}=\frac{4 \pi}{\alpha} \int \rho(x) x \sinh (\alpha x) d x
$$

then the potential outside the smearing radius $R$ remains the same as for the 
original point charge. For a uniform distribution inside a sphere of radius $R, \rho(r \leq R)=\frac{3}{4 \pi R^{3}}$, obtain

$$
\begin{aligned}
& q(\alpha, R)_{\text {uniform }}=Q(a R) \\
& \begin{aligned}
Q(t) & =\frac{2 t^{3}}{3\left[e^{t}(t-1)+e^{-t}(t+1)\right]} \\
& =1-\frac{t^{2}}{10}+\frac{9 t^{4}}{1400}+\ldots \leq 1
\end{aligned}
\end{aligned}
$$

The renormalization property enables (Rosenfeld, 1993a,b) to follow the Onsager "smearing" procedure as developed for obtaining a lower bound for the potential energy of Coulomb systems (Onsager, 1939; Lieb \& Narnhofer, 1975; Rosenfeld, 1982a, 1985; Rosenfeld \& Gelbart, 1984; Rosenfeld \& Blum, 1986) and apply it to general Yukawa systems.

Consider classical mixtures consisting of $N_{i}$ positively charged, $Z_{i} e>0$, point particles of type $i$, interacting through the Yukawa pair potentials :

$$
\frac{\phi_{i j}(r)}{k_{B} T}=Z_{i} Z_{j} \Gamma \frac{e^{-\alpha r}}{r}
$$

obtain the following optimized Onsager type lower bound (denoted as $u_{O n s} Y u k$ )

$$
\frac{U}{\Gamma N k_{B} T}=\frac{u_{O n s Y u k}}{\Gamma}=\sum_{i} x_{i} u_{i}
$$

expressed as the sum of "self" (dependent only on each type $i$ ) terms:

$$
u_{i}=\frac{Z_{i}^{2}}{R_{i}} u_{0}\left(\alpha R_{i}\right)
$$

with the function $u_{0}(t)$ related to $Q(t)$,

$$
u_{0}(t)=\frac{3}{2 t^{2}} Q(t)(t+1) e^{-t}
$$

and where the smearing radii,$R_{i}$, are obtained from the solution of the following set of non-linear coupled algebraic equations:

$$
R_{i}^{3}=\frac{Z_{i} Q\left(\alpha R_{i}\right)}{\sum_{i} x_{i} Z_{i} Q\left(\alpha R_{i}\right)} \quad, i=1,2, \ldots
$$


The excess (over ideal gas) energy of the plasma as evaluated in the standard linear response approximation of the electrons screening effects is (Firey \& Ashcroft, 1977; Chabrier \& Ashcroft, 1990; Rosenfeld \& Ashcroft, 1979)

$$
\begin{aligned}
u_{\text {mix,lin.resp. }} & =\frac{n}{2} \sum_{i j} x_{i} x_{j} \int\left[g_{i j}(r)-1\right] \phi_{i j}(r) d^{3} r \\
& -\frac{1}{2}<Z^{2}>e^{2} \lim _{r=0}\left[\frac{1}{r}-\phi(r)\right] \\
\frac{\beta u_{m i x, l i n . r e s p .}}{\Gamma} & \geq \sum_{i} x_{i} \frac{Z_{i}^{2}}{R_{i}} u_{0, \text { lin.resp. }}\left(\alpha R_{i}\right)
\end{aligned}
$$

corresponding to replacing $\mathrm{u}_{0}(\mathrm{t})$ in (39) by

$$
u_{0, \text { lin.resp. }}(t)=u_{0}(t)-\frac{3}{2 t^{2}}-\frac{t}{2}=-\frac{9}{10}-\frac{18}{175} t^{2}+\ldots
$$

It should be noted that the functions $Q(t)$ and $u_{0, l i n . r e s p .}(t)$ are even functions of their argument so that the Onsager bound for (41a) is an even function of $\alpha$. Thus, for small values of $\alpha$ the Onsager bound predicts the following expansion for the linear-response excess free energy:

$$
\frac{F(\Gamma, \alpha)}{\Gamma}=-\frac{9}{10}-\frac{18}{175} \alpha^{2}+O\left(\alpha^{4}\right)
$$

It is interesting to note here that the best fits (Hansen, 1975; Young, Corey, and DeWitt, 1991) to simulations data yield

$$
\frac{F(\Gamma, \alpha)}{\Gamma}=-\frac{8.992}{10}-\frac{18.165}{175} \alpha^{2}+\ldots
$$

for large values of $\Gamma$, in excellent agreement with the bound (43). The weak screening expansion for the Onsager bound for the mixture (eqs.(37)-(40)) is given by

$$
\begin{aligned}
u_{m i x, l i n . r e s p .} & =-0.9<Z^{5 / 3}><Z>^{1 / 3} \\
& +A_{\text {Non.Lin. }} \alpha^{2}+O\left(\alpha^{4}\right)
\end{aligned}
$$

where the first term is the standard linear-mixing (ion-sphere) result for unscreened plasmas, and 


$$
\begin{aligned}
& A_{\text {Non.Lin. }}=-\frac{\left\langle Z^{7 / 3}\right\rangle}{\langle Z\rangle^{1 / 3}}\left\{\frac{18}{175}+\frac{3}{100}\left[1-\frac{\left\langle Z^{5 / 3}\right\rangle^{2}}{\left\langle Z^{7 / 3}\right\rangle\langle Z\rangle}\right]\right. \\
& \left.+\frac{1}{100}\left[\frac{<Z^{5 / 3}>^{2}}{<Z^{7 / 3}><Z>}-\frac{<Z^{5 / 3}><Z>^{2 / 3}}{<Z^{7 / 3}>}\right]\right\}
\end{aligned}
$$

This expansion is in agreement only with general trends among the coefficients in a fit (Hubbard \& DeWitt, 1985) to simulations data which, as it stands, can not be compared in full detail with the present result. To obtain $A_{\text {Non.Lin }}$ we solve eqs.(40) to leading order :

$$
\begin{aligned}
R_{i}=\left(\frac{Z_{i}}{<Z>}\right)^{1 / 3}\left\{1-\frac{\alpha^{2}}{30}\left[\left(\frac{Z_{i}}{<Z>}\right)^{2 / 3}-\left(\frac{\left\langle Z^{5 / 3}\right\rangle}{<Z>^{2 / 3}<Z>}\right)\right]\right. \\
\left.+O\left(\alpha^{4}\right)\right\}
\end{aligned}
$$

The Onsager bound is the exact leading term in the strong coupling expansion for the fluid potential energy (Rosenfeld, 1985, 1986b, 1988). To the extent that its scaling properties apply also for arbitrary values of the coupling parameter, this strong coupling limit suggests the following general scaling approximation ("non-linear" mixing rule, because of eq.40) for repulsive-Yukawa fluids:

$$
\begin{aligned}
& u_{m i x}=\sum_{i} x_{i} u\left(\Gamma_{i}, \alpha_{i}\right) \\
& f_{m i x}=\sum_{i} x_{i} f\left(\Gamma_{i}, \alpha_{i}\right) \\
& \Gamma_{i}=\Gamma \frac{Z_{i}^{2}}{R_{i}}, \alpha_{i}=\alpha R_{i}
\end{aligned}
$$

where $u_{m i x}$ and $u$ refere to the mixture and to the one component energies (per particle, in temperature units), respectively, and $f_{m i x}$ and $f$ refere similarly to the corresponding excess free energies, and where eqs.(40) are used in (48b).

The Onsager exact energy bound for charged Yukawa mixtures and the corresponding non-linear mixing rule have a simple physical meaning which is revealed by treating the "Yukawa" problem in "Coulomb" language. In the standard linear-response (Firey \& Ashcroft, 1977; Chabrier \& Ashcroft, 1990) treatment for point ions in (e.g.) a nearly degenerate Fermi sea of electrons, it is assumed that the electron number density varies in space according to (let $e=1$ ) 


$$
\rho_{e}(\vec{r})=<\rho_{e}>+\Delta \rho_{e}(\vec{r})=n<Z>+\frac{\alpha^{2}}{4 \pi} \Phi(\vec{r})
$$

where $\Phi(\vec{r})$ is the electrostatic Coulomb potential at the point $r$ in space, and (Dharma-wardana \& Taylor, 1981; Dandrea, Ashcroft, and Carlsson, 1986) $\alpha$ is related to the Fermi function $I_{1 / 2}$. Let $U_{P}$ be the total electrostatic potential energy (relative to the infinite self energy of the point charges) of this electro-neutral system, and let $U_{K}$ be the non-ideal kinetic energy of the electrons in the linear response leading order. Their sum,

$$
U_{P}+U_{K}=\frac{1}{8 \pi} \int\left(|\vec{\nabla} \Phi(\vec{r})|^{2}+\alpha^{2} \Phi^{2}(\vec{r})\right) d V
$$

satisfies the following electrostatic inequality (Rosenfeld, 1991a,b, 1993b):

$$
U_{P}+U_{K} \geq \sum_{i} N_{i} u_{O A, i}=U_{O T F}
$$

where

$$
U_{O A, i}=\frac{1}{8 \pi} \int\left(|\vec{\nabla} \Phi(\vec{r})|^{2}+\alpha^{2} \Phi^{2}(\vec{r})\right) d v_{i}
$$

The integral $\int \ldots d v_{i}$ is over the volumes,$v_{i}$, of individual, confined, isolated, spherical, and neutral "Onsager-atoms" composed of a central point charge $Z_{i}$ and an electron cloud of number density (49) and radius $R_{i}$.

Using the definition of $Q(t)$ in Eqs.35 the electron density at the surface can be written as

$$
\rho_{e}\left(R_{i}\right)=\frac{Z_{i} Q\left(\alpha R_{i}\right)}{\left(\frac{4 \pi R_{i}^{3}}{3}\right)}
$$

The optimized bound (51) subject to the condition of total charge neutrality, $\sum_{i} x_{i} R_{i}^{3}=1$, yields the bound (41b). Comparison of (40) with (53) reveals its true physical meaning: it is the condition of constant (independent of $i$ ) electron surface density, i.e. constant surface electrostatic potential. This is of course the expected result on physical grounds, and which is missing in the linear rule. In view of the dominance of the electrons contribution to the total pressure of the plasma, which is determined for Thomas-Fermi theory 
by their density at the surface of the confined atom, the condition (40) also corresponds to the well known "volume additivity rule" for Thomas -Fermi mixing of elements (More, Warren, Young, and Zimmerman, 1988),

$$
v(P, T)=\sum_{i} x_{i} v_{i}(P, T)
$$

namely combining specific-volumes, $\mathrm{v}=\frac{V}{N}, \mathrm{v}_{i}=\frac{V_{i}}{N_{i}}$, at same pressure and temperature.

Using the non-linear mixing rule (48) obtain the following result for the zero separation screening potential of the Yukawa system

$$
H i j(0)=f\left(\Gamma_{i}, \alpha_{i}\right)+f\left(\Gamma_{j}, \alpha_{j}\right)-f\left(\Gamma_{i+j}, \alpha_{i+j}\right)
$$

where $\Gamma_{i+j}=\frac{\Gamma Z_{i+j}^{2}}{R_{i+j}}, \alpha_{i+j}=\alpha R_{i+j}$, and where $Z_{i+j}=Z_{i}+Z_{j}$. In the asymptotic strong coupling limit obtain

$$
\begin{aligned}
& \frac{H_{O M, i j}(0)}{\Gamma}=-\frac{9}{10}\left(\frac{Z_{i}^{2}}{R_{i}}+\frac{Z_{j}^{2}}{R_{j}}-\frac{Z_{i+j}^{2}}{R_{i+j}}\right)-Z_{i} Z_{j} \alpha \\
& -\frac{18}{175} \alpha^{2}\left(Z_{i}^{2} R_{i}+Z_{j}^{2} R_{j}-\left(Z_{i}+Z_{j}\right)^{2} R_{i+j}\right)+\ldots
\end{aligned}
$$

i.e.

$$
\begin{aligned}
& \frac{H_{O M, i j}(0)}{\Gamma}=-\frac{9}{10}<Z>^{1 / 3}\left(Z_{i}^{5 / 3}+Z_{j}^{5 / 3}-\left(Z_{i}+Z_{j}\right)^{5 / 3}\right)-Z_{i} Z_{j} \alpha \\
& -\left(\frac{18}{175}+\frac{3}{100}\right)\left(Z_{i}^{7 / 3}+Z_{j}^{7 / 3}-\left(Z_{i}+Z_{j}\right)^{7 / 3}\right)<Z>^{-1 / 3} \alpha^{2} \\
& +\frac{3}{100} \frac{\left\langle Z^{5 / 3}>\right.}{\left\langle Z>^{4 / 3}\right.}\left(Z_{i}^{5 / 3}+Z_{j}^{5 / 3}-\left(Z_{i}+Z_{j}\right)^{5 / 3}\right) \alpha^{2}+\ldots
\end{aligned}
$$

which for the one-component system takes the form (Rosenfeld, 1993b)

$$
\begin{aligned}
\frac{H_{O M}(0)}{\Gamma} & =-\frac{9}{10}\left(2-2^{5 / 3}\right)-\alpha \\
& +\left(\frac{18}{175}\left(2^{7 / 3}-2\right)+\frac{3}{100}\left(2^{7 / 3}-2^{5 / 3}\right)\right) \alpha^{2}+\ldots \\
& =-1.05732-\alpha+0.36860 \alpha^{2}+\ldots
\end{aligned}
$$

The present Eq. 58 corrects a small error in the original paper. Note however that in the physics context of the linear response for electron screening, 
the bare interaction between the ions is Coulomb, so that the "linear response" screening potential (Stein, shalitin, and Rosenfeld, 1988), for a pair of ions in the electron screened plasma, is an even function of $\alpha$.

$$
\begin{aligned}
H_{i j}(0) & =H_{i j}^{Y u k a w a}(0)+\left(\frac{Z_{i} Z_{j}}{r}-\phi_{i j}(r)\right)_{r=0} \\
& =H_{i j}^{Y u k a w a}(0)+Z_{i} Z_{j} \alpha
\end{aligned}
$$

\subsection{Relation of the Onsager molecule theory to the data obtained by simulations results and to its analysis}

\subsubsection{Concerning the simulation data}

Jancovici (1977) obtained his original expression

$$
\frac{H(0)}{\Gamma}=1.0531+2.2931 \Gamma^{-3 / 4}-\frac{(2.35+0.5551 \ln \Gamma)}{\Gamma}
$$

by using the "linear law" approximation for the excess (over ideal gas contribution) free energy of plasma mixtures, $F_{m i x}^{e x}$. This expression was obtained with a fit (DeWitt, 1976) to the best available simulation data at the time. With a most accurate fit to the best presently available data (Young, COrey, and DeWitt, 1991), in the range $(\Gamma>1)$

$$
f_{0}(\Gamma)=-0.8992 \Gamma+1.8322 \Gamma^{0.3253}-0.268 \ln \Gamma-1.3693
$$

the updated Jancovici expression is ( for $\Gamma>1$ )

$$
\frac{H(0)}{\Gamma}=1.05638+0.99643 \Gamma^{-0.6747}-\frac{(1.05969+0.268 \ln \Gamma)}{\Gamma}
$$

It is interesting to note that the leading Madelung term for the energy, which is a free parameter in the fit to the simulation data, comes closer to our prediction of -0.9 as the accuracy of the simulations increases. Our asymptotic analysis predicts (Rosenfeld 1985, 1986b) an expansion of the type

$$
f 0(\Gamma)=-0.9 \Gamma+a_{1} \Gamma^{\theta}+\ldots
$$

where $\theta=0.5$ is the exact result for the HNC approximation.

The linear and non-linear mixing rules for the thermodynamics of plasma mixtures without and with screening, respectively, are exact asymptotic 
scaling predictions of the Onsager-molecule approach which hold to a high accuracy also at relatively low values of $\Gamma \sim 1$. Concerning the accuracy of the linear law it should be emphasized that it holds to about $0.1 \%$ in the strong coupling region for all available simulation and hypernetted-chain approximation results (DeWitt, Slattery, and Stringfellow, 1990, Iyetomi, Ogata, and Ichimaru, 1988, 1991; Brami, Hansen, and Joly, 1979; Rosenfeld, $1982 \mathrm{~b}$ ). The same is true (Rosenfeld, 1993b) for the non-linear mixing rule for screened plasmas in the context of the hypernetted-chain approximation (extensive simulations data not yet available).

We demonstrated (Rosenfeld 1988,1991a) that the OCP structure at finite $\Gamma$ can be calculated very accurately by employing only the leading term in the strong coupling expansion of the bridge function around the "ideal" Onsager state:

$$
B(r, \Gamma)=b(r) \Gamma+b_{1}(r) \Gamma^{\lambda}+\ldots
$$

Indeed (Rosenfeld, Levesque, and Weis, 1989), by using $B(r, \Gamma)=\Gamma b(r)$ (see eq.23) in the structure equations (3)-(5), the results for the structure functions and for the equation of state at large values of $\Gamma$ (say, $\Gamma>40$ ) are comparable to those obtained from the fits to the simulations presented by Iyetomi , Ogata, and Ichimaru (1992). The correction terms, probably led by a $\Gamma^{\lambda}$ term with $\lambda \sim 1 / 3$, contain the long ranged contributions to the otherwise short ranged $\left(B_{O M}(r>2)=0\right)$ leading term. These corrections are of the order of the entropic contributions to the free energies involved in eq.(2), which are relatively small in comparison to the corresponding potential energy terms in strong coupling. The most sound procedure for extracting the bridge function from the simulation results for $g(r)$ is to analyse the difference $\Delta B(r)=B(r)-\Gamma b(r)$, which is on the border of the statistical accuracy of the simulations. Eventhough this method was not followed, other numerical solutions (Poll, Ashcroft, and DeWitt, 1988; Iyetomi , Ogata, and Ichimaru, 1992) of the inverse scattering problem for the OCP structure, using the simulations data as input, compare very well with our asymptotic predictions (Rosenfeld, 1988; Rosenfeld, Levesque, and Weis, 1989). Note that the fit by Ietomi , Ogata, and Ichimaru (1992) leads to $\frac{c(0)}{r}=-1.406$ as the leading term, which compares with our prediction (eq.20) of -1.4. As already discussed (Rosenfeld, 1988), the Onsager molecule result for the bridge function and for its universal component is in accord with the demonstratively successful modified-HNC theory based on the empirical Percus-Yevick bridge functions for hard spheres (Rosenfeld \& Ashcroft, 1979), $\mathrm{B}_{P Y H S}(\mathrm{r}, \eta)$, and an imposed thermodynamic consistency 
for determining the free parameter , $\eta(\Gamma)$. The resulting $B_{P Y H S}(r, \Gamma)$ features the long range oscilatory behavior, yet $\frac{B_{P Y H S}(r, \Gamma)}{\Gamma} \rightarrow 0.2 \omega(r)$ for $\Gamma \rightarrow \infty$. Explicit expressions for the universal bridge functional for inhomogeneous fluids, and an updated view of the extent of universality of the bridge functions, was presented recently (Rosenfeld, 1993a).

Finally, consider the expansion

$$
\frac{H(r)}{\Gamma}=h_{o}-h_{1} r^{2}+h_{2} r^{4}+h_{3} r^{6} \ldots
$$

where the coefficient $h_{1}$ is known exactly, $h_{1}=0.25$, and define the function

$$
\begin{aligned}
h_{2}(r) & =\frac{H(r)-H(0)+0.25 \Gamma r^{2}}{\Gamma r^{4}} \\
& =h_{2}+h_{3} r^{2}+\ldots
\end{aligned}
$$

which according to the Onsager molecule asymptotic prediction (eq.22) is a slowly varying function in the range $r<2$. Note that the Onsager molecule asymptotic result features the exact coefficient of the $r^{2}$ term in eq.(65), as well as the exact second moment of the electric microfield distribution (Rosenfeld, 1989). The fit by Alastuey and Jancovici (1978) to the Hansen data (Hansen \& Baus, 1980), yielding $h_{2}=0.039$, and $h_{3}=-0.0043$, is in accord with our Onsager molecule prediction. We do not have at hand the raw simulation data of Ogata, Iyetomi, and Ichimaru (1991) for $\frac{H(r)}{\Gamma}$, but it was fitted by them in the range $R_{\min } \leq r \leq 2$ to an accuracy of $0.1 \%$ by the function $f(r)$

$$
f(r)=A-B r+\exp \left(C r^{1 / 2}-D\right) / r
$$

where for $5<\Gamma<180$

$$
\begin{aligned}
& A=1.356-0.0213 \ln (\Gamma) \\
& B=0.456-0.0130 \ln (\Gamma) \\
& C=9.29+0.79 \ln (\Gamma) \\
& D=14.83+1.31 \ln (\Gamma)
\end{aligned}
$$

We found out that the function $f(r)$, in the actual data range for $r$, can be fitted to $0.05 \%$ by the expansion (65) with 3 free coefficients (i.e. upto the $r^{6}$ term), for all values of $\Gamma>\sim 70$, with results in about $0.3 \%$ agreement with the original Alastuey-Jancovici extrapolation for $H(0)$. This also 
shows that the functional form of $f(r)$ as chosen by Ogata, Iyetomi, and Ichimaru (1991), with 4 free coefficients, is inferior to the Alastuey-Jancovici form with 3 coefficients. Increasing the number of free coefficients to 5 almost does not affect the result of the extrapolation for these relatively large values of $\Gamma$. The resulting function $h_{0}(\Gamma)$ decreases with $\Gamma$ towords the asymptotic limit of 1.05732 , while the function $h_{2}(r)$ gradually increases towards the predicted Onsager molecule result. For $\Gamma=10$, however, only with 5 coefficients it is possible to fit the function $f(r)$ to better than $0.1 \%$, and the result features a negative coefficient $h_{2}$. This last result, and the gradual change in the value of $h_{2}$, from its asymptotic value of about 0.038 to zero (and even negative values) are in qualitative agreement with the direct simulation results of Ogata, Iyetomi, and Ichimaru (1991) for the coefficient $h_{2}$, as also discussed below.

\subsubsection{Concerning the data analysis of Ogata, Iyetomi, and Ichimaru}

Ogata, Iyetomi, and Ichimaru (1991) and Ichimaru \& Ogata (1990) employ the following methodology: they estimated the coefficient $h_{2}$ by the Monte Carlo method. They find that the computed values (which are smaller in magnitude than the extent of errors) are an order of magnitude smaller than $h_{1}$. As a result they decide to truncate the expansion at the second term with $h_{1}=0.25$, and obtain the extrapolated screening potential in the follwing form :

$$
\begin{aligned}
& \frac{H(r)}{\Gamma}=h_{0}-h_{1} r^{2} \quad \text { for } \quad r \leq r_{0} \\
& \frac{H(r)}{\Gamma}=f(r) \quad \text { for } \quad r \geq r_{0}
\end{aligned}
$$

The remaining two unknown parameters $h_{0}, r_{0}$ are determined from the requirement that the function and its first derivative are continuous at $r_{0}$ :

$$
\begin{aligned}
-0.5 r_{0} & =f^{\prime}\left(r_{0}\right) \\
h_{0}-0.25 r_{0}^{2} & =f\left(r_{0}\right)
\end{aligned}
$$

Since $f(r)$ is dominated by the linear part at $r_{0}$, Ogata, Iyetomi, and Ichimaru (1991) obtain 


$$
\begin{aligned}
& r_{0}=2 B=0.912-0.026 \ln (\Gamma) \\
& h_{0}=A-B^{2}=1.148-0.0094 \ln (\Gamma)-0.00017[\ln (\Gamma)]^{2}
\end{aligned}
$$

Near freezing, for example at $\Gamma=160$, corresponding to their Figure $1 \mathrm{~b}$, they obtain $r_{0}=0.780, h_{0}=1.095$. Jancovici's result $(1977), h_{0}=1.071$, is $2.2 \%$ smaller. Ogata, Iyetomi, and Ichimaru (1991) estimate the error , in their $H(0)=h_{0}$ so extrapolated, to stay on the order of $0.1 \%$.

This analysis by Ogata, Iyetomi, and Ichimaru (1991) is wrong. It is based on incorrect logic and incorrect methodology : (1) In strong coupling, e.g. $\Gamma=160$, the value of $r_{0}$ (e.g. $\left.r_{0}=0.78\right)$ is much smaller than $R_{\text {min }}$ (e.g. $R_{\min } \sim 1.15$ ). Thus, the connection point for the extrapolation is beyond the actual data points, making the procedure dependent on the choice of fitting function for the data. The obvious next step is to check what happens with the addition of extra terms. Indeed, keeping the term $h_{2}$, and repeating the procedure it is found (Rosenfeld, 1992a) that the connection point is now inside the data range, and the results are in $0.3 \%$ agreement with Alastuey and Jancovici. (2) Even if the coefficient $h_{2}$ is an order of magnitude smaller than $h_{1}$ its effect on the extrapolated $h_{0}$ can be of order of $1 \%$ (e.g. with $r_{0} \sim 1$ and $h_{2} \sim h_{1} / 25 \sim 0.01$ ). More specifically, observe their equations $\mathrm{A} 1, \mathrm{~A} 2$ and Table 4 . They write $h_{2}$ in the general form $\Gamma h_{2}=A-\Gamma^{2} / 32$, so that if $\Gamma h_{2}$ is close to zero then $A$ is close to $\Gamma^{2} / 32$. Since, for large $\Gamma, A$ is a large number for which one needs high accuracy, then it is indeed a good idea to do, as they did, and monitor the quantity " $u$ " in their eq.A2, which should provide the indication for the accuracy of the calculation for $A$. Now observe their Table 4 to find (e.g.) for $\Gamma=80: u=0.9910 \pm 0.000089$. in other words, the statistical accuracy is indeed very high, but the result for " $u$ " is $1 \%$ off the exact value $" u "=1$ in all the cases in Table 4. Thus, maybe the statistical error for $A$ is high and indicates \pm 0.01 for $h_{2}$, but on the basis of the "litmus test" via the quantity " $u$ " (as indeed intended and presented by them), one expects at least a similar error of about $1 \%$ for $A$, which means an error of about $\Gamma / 3200$ for $h_{2}$, i.e. 0.05 for $\Gamma=160$. Thus their claim $h_{2}=0.00 \pm 0.01$ is may be correct for $\Gamma=10$ but not for $\Gamma=160$. In addition to this expected systematic error, their data point for $\Gamma=160$ is: $h_{2}=0.063 \pm 0.133$, which at its maximum is of the order expected by the Onsager molecule theory. Such a possible gradual transition in the behavior of $h_{2}$ is in accord with our analysis ,above, of the simulation data as embodied by their function $f(r)$.

Ogata, Iyetomi, Ichimaru, and Van Horn $(1992,1993)$ find deviations from 
the linear mixing rule which seem to justify their results for $H(0)$ based on $h_{2}=0$. A closer look at their data reveals however that again, as above, they project $\Gamma \sim 10$ results to much stronger couplings. The significant data for the purpose of calculating $H(0)$ is for very dilute $\left(\left(x_{2} \sim 0.01\right)\right.$ mixture of larger charge $\left.\left(Z_{2}>1\right)\right)$ in a solvent of unit charge. The effective coupling for these relevant mixtures in the simulations of Ogata, Iyetomi, Ichimaru, and Van Horn $(1992,1993)$ are relatively weak (i.e. $\Gamma \sim 10)$. Moreover, their results for a "negative" deviation from the linear mixing rule lead to unphysical crystalization diagrams (Mochkovitch, these Proceedings), and contradict more accurate simulations by DeWitt \& Slattery (1993) which find a small positive deviation from the linear mixing rule.

In their analysis of their simulation data for mixtures, Ogata, Iyetomi, and Ichimaru (1991) do not consider the prefactor $G$, and thus effectively use the scaling (32a) with $G=1$. On the basis of the high accuracy of the linear law it is expected that scaling relations between the OCP and mixtures, which are similar to the linear law and are likewise based on the "Onsager molecule" (ion-sphere) limit, to be of similar high accuracy (e.g. better than the $0.3 \%$ which is the uncertainty in the OCP results). The additional systematic deviation introduced for unlike ions $(i \neq j)$ by the assumption that $G=1$, which is the assumption made by Ogata, Iyetomi, and Ichimaru can be estimated using (32b) by the deviations of $G_{0}$ from unity. Thus the results $G_{0}-1=0.999,0.993$ for $s=4 / 3,2$, respectively, justify the scaling relation used by them for Carbon/Oxygen and (somewhat less) Hydrogen/Helium mixtures. For larger charge ratios, however, significant deviations from the assumption $G=1$ will occur as, e.g. $G_{0}-1 \sim 0.03,0.06$ for $s=5,10$, respectively. In later work Ogata, Ichimaru, and Van Horn $(1991,1993)$ and Ogata, Iyetomi, Ichimaru, and Van Horn (1992) they use for the mixture the same incorrect extrapolation method as for the OCP, assuming the ion-sphere scaling via $d_{i j}$ for the function $f(r)$, again without regard to the short range correcting function $G(r)$. The raw simulations data of Ogata, Iyetomi, Ichimaru, and Van Horn agrees very well, however, with our Onsager molecule analysis.

For the unscreened OCP at $\Gamma=44.9$ Ichimaru and Ogata (1991) obtain $H(0) / \Gamma=1.110$ in contrast to the Jancovici type extrapolation (Rosenfeld, 1992a) $H(0) / \Gamma=1.088$. Considering the contribution of the electron response then the Ichimaru-Ogata result for $\alpha=0.3125$, namely $\delta$ $=1.110-0.825=0.285$, is similar to the corresponding alternative extrapolation result (Rosenfeld, 1992a) $\delta=1.088-0.800=0.288$, because the value of $h_{2}$ changes little, for this small value of $\alpha=0.3125$, from the 
$\alpha=0$ case. Ichimaru and Ogata compare their value of $\delta$ with another estimate, which they proposed earlier, given by (their eq.11)

$$
\delta=1.057\left(1-e^{-\alpha}\right)=1.057 \alpha-0.5285 \alpha^{2}+\ldots
$$

which gives $\delta=0.278$ for $\alpha=0.3125$. This value also compares well with the Onsager molecule estimate (eq.58) $\delta=\alpha-0.36860 \alpha^{2}+\ldots=0.277$. It is not clear what is the origin of eq.72, but it seems to be a fitting function. In any case, it should be noted that eq.(72) is analytically different (and for larger values of $\alpha$ also numerically different) from the Onsager molecule prediction eq. 58 which is derived from basic physics.

\subsection{Conclusion}

The present analysis finds excellent agreement between the simulations data for the equation of state and for the screening potentials of strongly coupled plasmas $(\Gamma>\sim 70)$, in accordance with the "Onsager molecule" asymptotic limit. The Alastuey-Jancovici-type extrapolation of the highly accurate simulations data of Ogata, Iyetomi, and Ichimaru (1991) for the OCP screening potential, together with the correct "Onsager molecule" scaling (Eq.32) provide the short range screening potentials of strongly coupled plasmas to an accuracy of about $0.3 \%$, in agreement with the linear law and equation of state data. The extended Jancovici formula (eq.55) for the Columb and Yukawa OCP agrees very well with the present extrapolation of the data of Ichimaru and Ogata (1991).

The inevitable conclusion from the present analysis is that the calculations of the enhancement factors for nuclear reaction rates of Ogata, Iyetomi, and Ichimaru (1991) and the calculations of the short range bridge functions of Iyetomi, Ogata, and Ichimaru (1992), which are all based on incorrect analysis of the short range screening potential for strongly coupled plasmas, should be revised. In particular, the new data analysis should take into account the gradual change of the coefficient $h_{2}(\Gamma)$ from its Onsager $\Gamma \rightarrow$ $\infty$ limit value of about 0.038 , to values near zero for $\Gamma \sim 20$ and to possibly negative values for $\Gamma<\sim 10$.

\section{Acknowledgements}

I thank Hugh DeWitt for bringing to my attention the papers by Ichimaru and coworkers, and for sending valueable material. Interesting discussions with Gilles Chabrier, Hugh DeWitt, Jordi Isern and Hugh Van Horn are acknowledged with gratitude. This work was supported by The Basic Re- 
search Foundation administered by The Israel Academy of Sciences and Humanities under Grant No.492/92.

\section{References}

Aers G.C. and Dharma- wardana M.W.C., Phys.Rev.A29, 2734, (1984)

Alastuey A. and Jancovici B., Ap.J. 226, 1034 , (1978)

Brami B., Hansen J.P., and Joly F., Physica 905A, 505, (1979)

Chabrier G. and Ashcroft N.W., Phys.Rev.A42, 2284, (1990)

Dandrea R.G., Ashcroft N.W., and Carlsson A.E., Phys.Rev.B 34 , 2097, (1986)

Dharma-wardana M.W.C. and Taylor R., J.Phys.C14, 629, (1981)

DeWitt H.E., Graboske H.C., and Cooper M.S., Ap.J. 181 , 439, (1973)

DeWitt H.E., Phys.Rev.A14,1290, (1976)

DeWitt H.E., Slattery W.L., and Stringfellow G.S., in "Strongly Coupled Plasma Physics", edited by S.Ichimaru, North-Holland/Yamada Science Foundation, Amsterdam, 1990, page 635 .

DeWitt H.E. and Slattery W.L., privat communication, (1993)

Firey B. and Ashcroft N.W., Phys.Rev.A15 , 2072, (1977)

Hansen J.P., J.Physique.36, L-133, (1975)

Hansen J.P., Torrie G.M., and Vieillefosse P., Phys.Rev.A16, 2153, (1977)

Hansen J.P. and Baus M., Phys.Rep.59, 1, (1980)

Hoover W.G. and Poirer J.C., J.Chem.Phys.37, 1041, (1962)

Hubbard W.B. and DeWitt H.E., Astrophys.J. 290, 388, (1985)

Jancovici B., J.Stat.Phys.17, 357, (1977)

Ichimaru S., Rev.Mod.Phys. 54, 1017, (1982)

Ichimaru S. ,Iyetomi H., and Tanaka S., Phys.Rep. 149, 91, (1987)

Ichimaru S., Ed., Strongly Coupled Plasma Physics", North- Holland/Yamada Science Foundation, Amsterdam, 1990

Ichimaru S. and Ogata S., in "Strongly Coupled Plasma Physics", edited by S.Ichimaru, North-Holland/Yamada Science Foundation, Amsterdam, 1990, page 101.

Ichimaru S. and Ogata S., Ap.J.374,647, (1991)

Ichimaru S., Rev.Mod.Phys.65, 255, (1993)

Itoh N., Totsuji H., Ichimaru S., Ap.J.218, 477, (1978)

Itoh N., Totsuji H., Ichimaru S., and DeWitt H.E., Ap.J.234, 1079, (1979)

Iyetomi H., Ogata S., and Ichimaru S., Ap.J.334, L17, (1988)

Iyetomi H., Ogata S., and Ichimaru S., in US-Japan Workshop on Nuclear Fusion in Dense Plasmas, Institute for Fusion Studies, The University of Texas, Austin. Preliminary Abstracts, Edited by S.Ichimaru and T.Tajima, Oct. 1991

Iyetomi H., Ogata S., and Ichimaru S., Phys.Rev.A46 ,1051, (1992)

Lieb E.L. and Narnhofer H., J.Stat.Phys.12, 291, (1975)

More R.M. , Warren K.H., Young D.A., and Zimmerman G.B., Phys.Fluids 31, 3059, (1988)

Ogata S., Iyetomi H., and Ichimaru S., in "Strongly Coupled Plasma Physics", edited by S.Ichimaru, North-Holland/Yamada Science Foundation, Amsterdam, 1990, page 59

Ogata S., Iyetomi H., and Ichimaru S., Ap.J.372, 259, (1991) 
Ogata S., Ichimaru S., and Van Horn H.M., in US-Japan Workshop on Nuclear Fusion in Dense Plasmas, Institute for Fusion Studies, The University of Texas, Austin. Preliminary Abstracts, Edited by S.Ichimaru and T.Tajima, Oct.1991

Ogata S., Iyetomi H., Ichimaru S., and Van Horn H.M. , in proceedings of the International Conference on the Physics of Strongly Coupled Plasmas, Rochester, New-York, USA, August 17 - 21,1992 (University of Rochester Press, in print, edited by $H$. VanHorn and S. Ichimaru);

Ogata S., Ichimaru S., and Van Horn H.M. , preprint , 1993

Onsager L. , J.Phys.Chem. 43, 189, (1939)

Poll P.D., Ashcroft N.W., and DeWitt H.E., Phys.Rev.A37 , 1672, (1988)

Rogers F.J.and DeWitt H.E., Eds., Strongly Coupled Plasma Physics", Plenum, New York, 1987

Rosenfeld Y. and Ashcroft N.W., Phys.Rev.A20 ,1208, (1979)

Rosenfeld Y., Phys. Rev. Letters, 44, 146, (1980a)

Rosenfeld Y., J. Physique. 41 , C2 -77, (1980b)

Rosenfeld Y., Phys.Rev.A25,1206(1982a)

Rosenfeld Y., Phys.Rev.A26, 3622, (1982b)

Rosenfeld Y. and Gelbart W.M., J. Chem. Phys., 81, 4574, (1984)

Rosenfeld Y., Phys.Rev.A32, 1834(1985)

Rosenfeld Y., J. Stat. Phys. 42, 437, (1986a)

Rosenfeld Y., Phys.Rev.A 33, 2025, (1986b)

Rosenfeld Y. and Blum L., J. Chem.Phys. 85 , 1556, (1986)

Rosenfeld Y., Phys.Rev.A35, 938, (1987a)

Rosenfeld Y., in "Strongly Coupled Plasma Physics", edited by F.J.Rogers and H.E.DeWitt, Plenum, New York, 1987b.

Rosenfeld Y., Phys.Rev.A37, 3403, (1988)

Rosenfeld Y., Levesque D., and Weis J.J., Phys.Rev.A 39 , 3079, (1989)

Rosenfeld Y., Phys.Rev.A40,1137, (1989)

Rosenfeld Y., J.Chem.Phys. 93, 4305, (1990)

Rosenfeld Y.,in High-Pressure Equations of State : Theory and Applications, edited by S. Eliezer and R. Rici, Italian Physical Society International School of Physics Enrico Fermi, Course CXIII ,1989, North- Holland, Amsterdam, (1991a)

Rosenfeld Y., Phys.Rev.A43, 6526, (1991b)

Rosenfeld Y., Phys.Rev.A46, 1059, (1992a)

Rosenfeld Y., A46, 4922, (1992b)

Rosenfeld Y., J.Chem.Phys.98,8126, (1993a)

Rosenfeld Y., Phys.Rev.E47, 2676, (1993b)

Rowlinson J.S. and Widom B., Molecular Theory of Capilarity (Clarendon, Oxford, 1982)

Rowlinson J.S. , Physica A156, 15, (1989)

Salpeter E.E., Aust.J.Phys.7, 373, (1954)

Salpeter E.E. and Van Horn H.M.,Ap.J. 155, 183, (1969)

Stein J., Shalitin D., and Rosenfeld Y., Phys.Rev.A37 , 4854, (1988)

Widom B., J.Chem.Phys.39, 2808, (1963)

Young D.A., Corey E.M., and DeWitt H.E., Phys.Rev.A44, 6508, (1991) 